\title{
Arthroscopic management of temporomandibular closed lock
}

\author{
Hizuru Miyamoto, DDS* \\ Hideaki Sakashita, DDS, PhD† \\ Masaru Miyata, DDS, $\mathrm{PhD} \ddagger$ \\ Alastair N. Goss, DDSc, FRACDS(OMS), FICD $\$$ \\ Koichi Okabe, DDS, PhD, \\ Yuuko Miyaji, DDS, \\ Kuniko Sakuma, DDS,
}

\begin{abstract}
Sixty-three consecutive patients with painful closed lock involving 83 temporomandibular joints were treated by surgery and followed up for at least 24 months. Prior to surgical treatment all had imaging to demonstrate that the cause of their painful jaw locking was an anterior displaced disc without reduction. All patients had received at least three months non-surgical treatment without response. The arthroscopic surgery consisted of release of adhesions, manipulation and placement of $25 \mathrm{mg}$ hyaluronic acid into the joint space. No significant surgical complications occurred. Postoperative treatment involved physical therapy and stabilization splints. At 24 months after surgery 57 patients (90.5 per cent) had no pain and mouth opening had improved from preoperative $27.2 \pm 5.4 \mathrm{~mm}$ to $44.4 \pm 4.1 \mathrm{~mm}(p<0.001)$. This study shows that arthroscopic surgery with continuing non-surgical therapy is a highly effective treatment for painful closed lock.
\end{abstract}

Key words: Temporomandibular joint, closed lock, arthroscopic management.

(Received for publication June 1997. Revised August 1997. Accepted August 1997.)

\section{Introduction}

Temporomandibular joint (TMJ) closed lock or Stage III internal derangement is a painful condition which results in a major disturbance of masticatory function. ${ }^{1}$ Initial treatment should be non-surgical, involving reassurance, physical therapy, pharmacological therapy and occlusal appliance therapy. ${ }^{2}$ None of these treatments is universally successful and in non-responsive cases surgery should be considered. ${ }^{3}$ Arthroscopic surgery involves minimal invasion of the joint but allows direct vision of the intra-articular space. ${ }^{4}$

There have been several studies of the outcome of arthroscopic surgery. ${ }^{5-12}$ These studies can be criticized as having heterogeneous patient and intraarticular pathosis pools, short-term follow-up and poorly defined success criteria. The American Association of Oral and Maxillofacial Surgeons, ${ }^{13}$ the American Society of TMJ Surgeons ${ }^{14}$ and the International Association of Oral and Maxillofacial Surgeons $\mathrm{s}^{3,15,16}$ have provided practical guidance for the evaluation of surgical management of TMJ problems.

This study evaluated the long-term effectiveness of a standardized arthroscopic treatment regime for a consecutive population of Japanese patients with Stage III internal derangement.

^Staff, Department of Dentistry and Oral Surgery, Ishikawa Prefectural Central Hospital; and Visiting Research Fellow, Oral and Maxillofacial Surgery Unit, Department of Dentistry, The University of Adelaide.

†Chief, Department of Dentistry and Oral Surgery, Ishikawa Prefectural Central Hospital.

¥Assistant Chief, Department of Dentistry and Oral Surgery, Ishikawa Prefectural Cental Hospital.

§Professor and Director, Oral and Maxillofacial Surgery Unit, Department of Dentistry, The University of Adelaide.

,Staff, Department of Dentistry and Oral Surgery, Ishikawa

Prefectural Central Hospital.

\section{Materials and methods}

Consecutive patients with Stage III internal derangement who presented to the Ishikawa Prefectural Central Hospital, Kanazawa, Japan, between December of 1991 and July of 1994 and who met the following criteria were included in this study: 

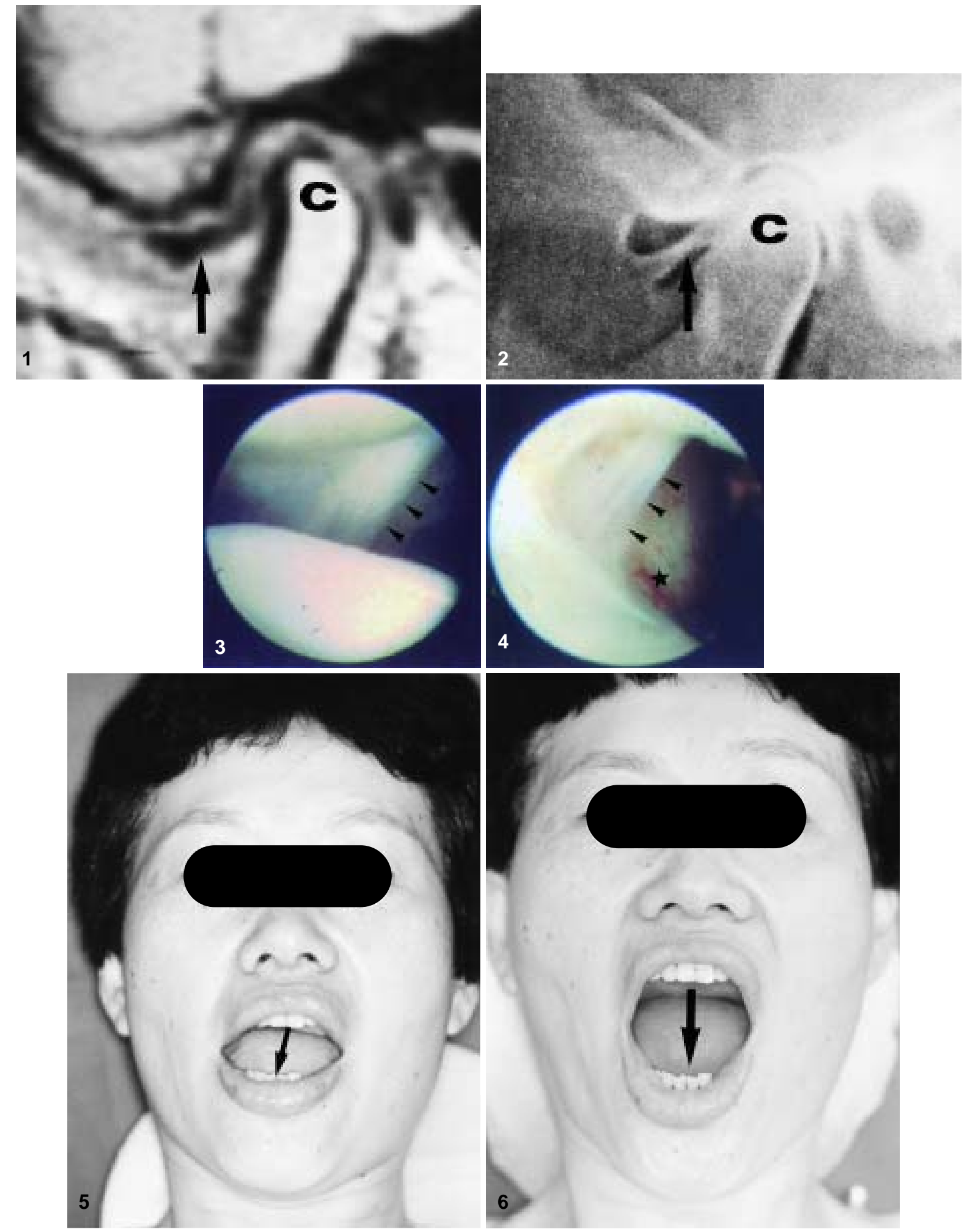

Fig. 1. - Magnetic resonance image (MRI) demonstrating a complete anterior disc displacement. (C: condyle; arrow: disc). Fig. 2. - Double contrast arthrogram demonstrating a complete anterior disc displacement. (C: condyle; arrow: disc). Fig. 3. - Arthroscopic findings of the superior joint space of the right side showing a mild adhesion (arrows).

Fig. 4. - Arthroscopic findings of the superior joint space of the left side showing adhesions (arrows) and synovitis (star). Fig. 5. - Preoperative view of patient with closed lock of the right side. The patient could open the jaw only $30 \mathrm{~mm}$ and the jaw deviated toward the right.

Fig. 6. - Postoperative view at 10 days of the same patient as Fig. 5. The patient could open the jaw $42 \mathrm{~mm}$ with no deviation. 
Table 1. Criteria of American Association of Oral and Maxillofacial Surgeons (1984) ${ }^{13}$

1. Pain that is absent or so mild, brief and infrequent as to be of no concern to the patient.

2. Range of motion greater than $35 \mathrm{~mm}$ for vertical and greater than $6 \mathrm{~mm}$ for protrusive and lateral excursions.

3. Regular diet which, at worst, avoids tough or hard foods. Patient must be no more than minimally inconvenienced by diet.

4. Radiographic changes in the joint. These are probably not important unless there are severe destructive changes.

5. Absence of significant complications.

1) Clinically reduced and painful maximum mouth opening;

2) MRI and/or a contrast arthrogram demonstration of anterior disc displacement without reduction (Fig. 1, 2);

3) Unsuccessful non-surgical treatment for at least three months prior to surgery.

Patients with evidence of major jaw trauma, systemic joint or muscle disease, and major jaw deformities were excluded from the study.

Under general anaesthesia, arthroscopic lysis and lavage with mandibular manipulation was performed using a miniscope set. After a double puncture arthroscopic examination of the superior joint space (Fig. 3, 4), adhesions were either detached using a blunt trocar or resected using an electric cautery or microscissors under direct vision, and lateral lysis was performed using a blunt trocar. ${ }^{10,16}$ The mandible was then manipulated. The joint space was irrigated with $400 \mathrm{~mL}$ of lactated Ringer's solution and instilled with $25 \mathrm{mg}$ of hyaluronate sodium. ${ }^{\star \star}$ Post-surgical treatment comprised of a full-coverage occlusal stabilization splint and physical therapy, which focused on muscle relaxation and posture. Three days after surgery, each patient began a training programme for maximum opening, protrusive and lateral movements.

Data on the pain, range of motion, regular diet and absence of complications, in accordance with the criteria of the American Association of Oral and Maxillofacial Surgeons (Table 1), ${ }^{13}$ were recorded. Patients were reviewed regularly with a final postoperative visit at a mean of 34.4 (range 24-56) months after arthroscopic surgery. Results were assessed using the following criteria: cases that satisfied all four criteria were rated as excellent, cases that satisfied three criteria were rated as good, and cases that satisfied less than three criteria were rated as unsatisfactory. For statistical analysis, repeated measures in one-way analysis of variance were used to compare maximum interincisal opening (MIO) between the preoperative and postoperative stages.

TStryker TMJ Mini-Scope Set. Stryker, USA

${ }^{\star \star A}$ Art ${ }^{\circledR}$. Seikagaku-kougyou, Japan.
Table 2. Patient data summary

\begin{tabular}{lc}
\hline Subject & Average age (years) \\
\hline 63 patients with 83 joints & $26.0 \pm 9.1$ \\
(6 males and 57 females) & (Range: 16 to 50$)$ \\
\hline
\end{tabular}

Table 3. Postoperative pain

\begin{tabular}{lc}
\hline & No. of patients \\
\hline No pain & $57(90.5 \%)$ \\
Reduced pain & $5(7.9 \%)$ \\
Pain the same & $1(1.6 \%)$ \\
\hline
\end{tabular}

All patients had severe or moderate preoperative pain.

Visual Analog Scale (0-100) was used.

Table 4. Maximum vertical mouth opening (mm)

\begin{tabular}{lc}
\hline Preoperative & Postoperative $^{\star}$ \\
\hline $27.2 \pm 5.4$ & $44.4 \pm 4.1$ \\
\hline
\end{tabular}

^Final postoperative visit at 34.4 (24-56) months. $\mathrm{p}<0.001$.

\section{Results}

A total of 63 patients (83 joints) who were followed up for at least 24 months after surgery were included in this study (Table 2). The postoperative level of pain is presented in Table 3. The pre- and postoperative MIO were compared and there was a significant improvement ( $\mathrm{p}<0.001$, Table 4, Fig. 5, 6 ). Only one patient, who had the same pain as preoperatively, was not able to open his mouth more than $35 \mathrm{~mm}$. No patient complained of difficulty in eating a normal diet. A slight and transient facial palsy of the temporal branch at the VIIth nerve occurred on the operated side in four patients (6.3 per cent). Normal nerve function returned in all four patients within three months. The success rate was excellent for 57 patients (90.5 per cent) and 77 joints (92.8 per cent); good for five patients (7.9 per cent) and five joints (6 per cent); and unsatisfactory for a single joint in one patient ( 1.6 per cent).

\section{Discussion}

This study showed that arthroscopic management was highly effective in the treatment of closed lock refractory to non-surgical treatment. The arthroscopic treatment involved a combination of procedures. First, the adhesions within the joint space were all detached under direct arthroscopic vision. This involved not only removal of all adhesions within the superior joint space, but also the release of the hypertrophic or tense lateral capsule. After lateral lysis, the mandible was then manipulated to ensure that there was a full range of movement. If there was not a full range of movement, then further arthroscopic release was performed. 
The joint space was then copiously irrigated to remove debris and inflammatory mediators. Finally, $25 \mathrm{mg}$ of hyaluronic acid was placed into the joint space. Following arthroscopic surgery, rigorous nonsurgical treatment was instituted. This treatment combination resulted in complete resolution of pain in 90.5 per cent of the cases and a full range of movement in 98.8 per cent of the cases.

The relative contributions of the various techniques used in the combination are difficult to define. Adhesions can only be treated by arthroscopic resection. Other studies ${ }^{6-11,17}$ using lysis and lavage alone have shown an 80-90 per cent success rate. Manipulation was used to confirm that a full range of movement could occur and if initially unsuccessful further arthroscopic release was indicated. Joint irrigation is an essential part of lysis and lavage to remove debris and to reduce pain-inducing inflammatory mediators. ${ }^{18}$

Hyaluronate sodium alone has been shown to reduce pain in internally deranged joints. ${ }^{19}$ Animal studies where osteoarthrosis was induced and then treated with hyaluronic acid showed a reversal of the osteoarthrotic process. ${ }^{20}$

Thus, on completion of the arthroscopic procedure, all joints were free of adhesions, had a good range of movement and inflammatory mediators were flushed out. The intra-articular space was then lubricated with hyaluronic acid. Hence the patients could cope with immediate non-surgical mobilization and splint therapy.

A minority of five patients, involving six joints, had persisting but reduced pain. One patient remained relatively unchanged. For these unsatisfactory patients either open operation ${ }^{21}$ or chronic pain management is indicated.

\section{References}

1. Wilkes $\mathrm{CH}$. Internal derangements of the temporomandibular joint: pathological variations. Arch Otolaryngol Head Neck Surg 1989;115:469-77.

2. Okeson JP. Rationale for non-surgical temporomandibular joint management. In: Stegenga B, de Bont LGM, eds. Management of temporomandibular joint degenerative disease: biological basis and treatment outcome. Berlin: Birkhauver Verlag, 1996.

3. Goss AN. Toward an international consensus on temporomandibular joint surgery. Int J Oral Maxillofac Surg 1993;22:7881.

4. American Association of Oral and Maxillofacial Surgeons. Position paper on TMJ arthroscopy. J Oral Maxillofac Surg 1993;50:Suppl 7.

5. Dimitroulis G, Dolwick MF. Temporomandibular disorders. Part 3. Surgical treatment. Aust Dent J 1996;41:16-20.
6. Indresano TA. Arthroscopic surgery of the temporomandibular joint; report of 64 patients with long-term follow-up. J Oral Maxillofac Surg 1989;47:439-41.

7. Montgomery M, Van Sickles J, Harms SE. Arthroscopic TMJ surgery; Effects on signs, symptoms and disc position. J Oral Maxillofac Surg 1989;47:1263-71.

8. Moses JJ, Poker ID. TMJ arthroscopy; An analysis of 237 patients. J Oral Maxillofac Surg 1989;47:790-4.

9. McCain JP, Sanders B, Koslin MG, Quint JD, Peters PB, Indresano TA. Temporomandibular joint arthroscopy: a 6-year multicenter retrospective study of 4,831 joints. JOral Maxillofac Surg 1992;50:926-30.

10. Miyamoto H, Sakashita H, Miyata M. Clinical study on arthroscopic surgery of the temporomandibular joint. Jpn J Diag/Oral Med 1993;6:265-9.

11. Kawakami T, Tsuzuki M, Shohara E, Takayama K, Ohkohchi N, Morimoto Y, Fujita H, Sugimura M. Evaluation of surgical treatment for internal derangement with closed lock of the temporomandibular joint: postoperative follow-up study of arthroscopic surgery. Jpn J Oral Maxillofac Surg 1995;41:780-4.

12. Chossegros C, Cheynet F, Gola R, Pauzie F, Arnaud R, Blanc JL. Clinical results of therapeutic treatment temporomandibular joint arthroscopy: a prospective study of 34 arthroscopies with prediscal section and retrodiscal coagulation. $\mathrm{Br}$ J Oral Maxillofac Surg 1996;34:504-7.

13. Dolwick MF. 1984 criteria for TMJ meniscus surgery. Chicago: Am Assoc Oral Maxillofac Surg, 1984:1-40.

14. Boering G, de Bont LGM, Stegenga B. Guidelines for diagnosis and management of temporomandibular disorders. Groningen: Am Soc TMJ Surg, 1990.

15. Holmlund AB. Surgery for TMJ internal derangement: evaluation of treatment outcome and criteria for success. Int J Oral Maxillofac Surg 1993;22:75-7.

16. Sanders B. Arthroscopic surgery of the temporomandibular joint: treatment of internal derangement with persistent closed lock. Oral Surg Oral Med Oral Pathol 1986;62:361-72.

17. White RD. Retrospective analysis of 100 consecutive surgical arthroscopies of the temporomandibular joint. J Oral Maxillofac Surg 1989;47:1014-21.

18. Sasaki K. Use of a balloon catheter in TMJ arthroscopic surgery for internal derangement of the temporomandibular joint. Jpn J Oral Maxillofac Surg 1996;42:1136-8.

19. Bertolami CN, Gay T, Clark G, Rendell J, Shetty V, Liu C, Swann DA. Use of sodium hyaluronate in treating temporomandibular joint disorders: a randomized double-blind, placebocontrolled clinical trial. J Oral Maxillofac Surg 1993;51:232-42.

20. Neo H, Ishimaru J-I, Kurita K, Goss AN. The effect of hyaluronic acid on temporomandibular joint osteoarthrosis: a animal study using sheep. J Oral Maxillofac Surg 1997;55:1114-9.

21. Trumpy IG, Lyberg T. Surgical treatment of internal derangement of the temporomandibular joint: long-term evaluation of three techniques. J Oral Maxillofac Surg 1995;53:740-6.

\section{Address for correspondence/reprints: Dr Hizuru Miyamoto, Visiting Research Fellow, Oral and Maxillofacial Surgery Unit, Department of Dentistry, The University of Adelaide, Adelaide, South Australia 5005.}

